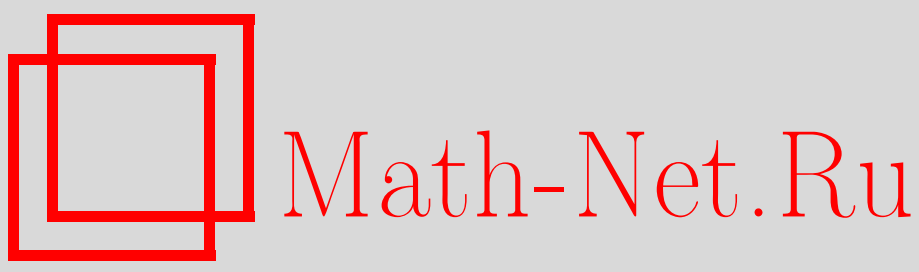

В. П. Михайлов, О существовании предельных значений на границе области решений полигармонического уравнения, Матем. сб., 1996, том 187, номер 11, 89-114

DOI: https://doi.org/10.4213/sm173

Использование Общероссийского математического портала Math-Net.Ru подразумевает, что вы прочитали и согласны с пользовательским соглашением http://www . mathnet.ru/rus/agreement

Параметры загрузки:

IP: 3.85 .7 .115

26 апреля 2023 г., 18:07:29 
УДК 517.95

\author{
В.П. Михайлов
}

\title{
О существовании предельных значений на границе области решений полигармонического уравнения
}

\footnotetext{
В работе установлены необходимые и достаточные условия существования пределов в среднем полигармонических функций в круге.

Библиографол: 2 названия.
}

Нас интересует вопрос о существовании предельных значений на границе области решений эллиптических уравнений. Задача рассматривается в самой простой ситуации: двумерный случай, область - круг, уравнение, предельными значениями решений на границе которого мы будем интересоваться, - полигармоническое, точнее $m$-гармоническое, $m \geqslant 1$ :

$$
\Delta^{m} u=0, \quad|x|<1 .
$$

Под предельным значением на границе $\{|x|=1\}$ непрерьвной в $\{|x|<1\}$ функции $u(x)=u(r, \varphi), x=\left(x_{1}, x_{2}\right)=(r \cos \varphi, r \sin \varphi), 0 \leqslant r<1$, будем понимать ее $L_{2}$-предельные значения: непрерьвная в круге $\{r<1\}$ функция $u(r, \varphi)$ имеет $L_{2}$-предел на гранище $\{r=1\}$, если существует функция $f(\varphi) \in L_{2}(0,2 \pi)$ такая, что

$$
\int_{0}^{2 \pi}(u(r, \varphi)-f(\varphi))^{2} d \varphi=\|u(r, \cdot)-f(\cdot)\|_{L_{2}}^{2} \rightarrow 0 \text { при } r \rightarrow 1 .
$$

Соответственно с этим функцию $u(r, \varphi)$ будем называть $L_{2}$-ограниченной, если

$$
\sup _{r<1}\|u(r, \cdot)\|_{L_{2}}<\infty
$$

Хорошо известны критерии сушествования $L_{2}$-предельных значений на границе у решений эллиптических уравнений второго порядка. Приведем два из них. В рассматриваемой ситуации (область - круг, и $m=1$ в (1)) они выглядят следуюшим образом. Первый критерий - теорема типа теоремы Рисса: для сушествования $L_{2}$-предела решения на границе $\{|x|=1\}$ необходимо и достаточно, чтобы это решение было $L_{2}$-ограниченным. Второй критерий - теорема типа теоремы Литлвуда-Пэли: для сушествования $L_{2}$-предела на границе решения необходимо и достаточно выполнение условия

$$
\int_{|x|<1}|\nabla u|^{2}(1-|x|) d x<\infty
$$


Эти утверждения, являясь критериями сушествования $L_{2}$-пределов у решений уравнения второго порядка, перестают быть необходимьми и достаточными условиями для решений уравнения более высокого порядка, $m>1$. При этом условие $L_{2}$-ограниченности остается, конечно, необходимьм условием существования $L_{2}$-предела, но перестает быть условием достаточньм. Условие же $(3)$, наоборот, является достаточным условием сушествования $L_{2}$-предела решения, но не является для этого необходимым. Достаточность условия (3) можно получить с помощью результатов работы [1], впрочем, для рассматриваемого случая ее легко получить и непосредственно. Приведем примеры, показьвающие недостаточность условия $L_{2}$-ограниченности и не необходимость условия $(3)$ для существования $L_{2}$-пределов на границе. Эти примеры можно найти среди бигармонических функций - решений уравнения (1) при $m=2$ (бигармоническая функция, конечно, является и $m$-гармонической при любом $m \geqslant 2$ ).

Прежде всего заметим, что при любой ограниченной числовой последовательности $\varepsilon_{1}, \ldots, \varepsilon_{n}, \ldots$ функция

$$
u(r, \varphi)=\left(1-r^{2}\right) \sum_{k=1}^{\infty} \varepsilon_{k} \sqrt{k} r^{k} \cos k \varphi, \quad r<1
$$

является бигармонической в круге $\{|x|<1\}=\{r<1\}$. Далее, так как

$$
\|u(r, \cdot)\|_{L_{2}}^{2}=\pi\left(1-r^{2}\right)^{2} \sum_{k=1}^{\infty} \varepsilon_{k}^{2} k r^{2 k}
$$

то для всех $r<1$

$$
\|u(r, \cdot)\|_{L_{2}}^{2} \leqslant \pi\left(1-r^{2}\right)^{2} M_{1}^{2} \sum_{k=1}^{\infty} k r^{2 k}=\pi M_{1}^{2} r^{2} \leqslant \pi M_{1}^{2}
$$

где $M_{1}=\sup _{k \geqslant 1}\left|\varepsilon_{k}\right|<\infty$, т.е. функция $u(r, \varphi)$ из (4) $L_{2}$-ограничена. Покажем, что в случае, когда $\varepsilon_{1}=\cdots=\varepsilon_{n}=\cdots=1$, функция $u(r, \varphi) L_{2}$-предела на границе не имеет. Прежде всего отметим, что в этом случае из (5) следует равенство

$$
\|u(r, \cdot)\|_{L_{2}}^{2}=\pi\left(1-r^{2}\right)^{2} \sum_{k=1}^{\infty} k r^{2 k}=\pi r^{2} \quad \text { для всех } r<1 .
$$

Далее, если существует $L_{2}$-предел $f(\varphi)$ на границе $\{r=1\}$ функции $u(r, \varphi)$, то в силу слабой сходимости

$$
\begin{gathered}
\int_{0}^{2 \pi} f(\varphi) d \varphi=\lim _{r \rightarrow 1} \int_{0}^{2 \pi} u(r, \varphi) d \varphi=0, \\
\int_{0}^{2 \pi} f(\varphi) \sin k \varphi d \varphi=0, \quad k \geqslant 1, \\
\int_{0}^{2 \pi} f(\varphi) \cos k \varphi d \varphi=\lim _{r \rightarrow 1}\left(1-r^{2}\right) \sqrt{k} r^{k}=0, \quad k \geqslant 1 .
\end{gathered}
$$


Это означает, что $f=0$, но этого быть не может в силу $(6): \lim _{r \rightarrow 1}\|u(r, \cdot)\|_{L_{2}}=$ $\sqrt{\pi} \neq 0$. Утверждение о недостаточности условия $L_{2}$-ограниченности для существования $L_{2}$-предела на границе установлено.

Покажем, что если последовательность $\varepsilon_{k}, k=1,2, \ldots$, стремится к нулю, $\varepsilon_{k} \rightarrow 0, k \rightarrow \infty$, то заданная формулой $(4)$ функция $u(r, \varphi)$ имеет нулевой $L_{2}$-предел на границе. Действительно, для произвольного $\varepsilon>0$ найдем $N=N_{\varepsilon}>0$ такое, что $\left|\varepsilon_{k}\right| \leqslant \varepsilon$ для всех $k \geqslant N$. В связи с этим из (5) получаем справедливое для всех $r<1$ неравенство

$$
\begin{aligned}
\|u(r, \cdot)\|_{L_{2}}^{2} & =\pi\left(1-r^{2}\right)^{2}\left(\sum_{k=1}^{N} \varepsilon_{k}^{2} k r^{2 k}+\sum_{k=N+1}^{\infty} \varepsilon_{k}^{2} k r^{2 k}\right) \\
& \leqslant \pi\left(1-r^{2}\right)^{2} M_{1}^{2} \frac{N(N+1)}{2}+\varepsilon^{2} \pi\left(1-r^{2}\right)^{2} \sum_{k=1}^{\infty} k r^{2 k} \\
& =\pi\left(1-r^{2}\right)^{2} \frac{M_{1}^{2} N(N+1)}{2}+\varepsilon^{2} \pi r^{2},
\end{aligned}
$$

из которого следует, что

$$
\|u(r, \cdot)\|_{L_{2}}^{2} \leqslant 2 \pi \varepsilon^{2} \text { для всех } r \geqslant r_{0},
$$

где $\left(1-r_{0}^{2}\right)^{2} \frac{M_{1}^{2} N(N+1)}{2}=\varepsilon^{2}$. Что и требовалось установить. Теперь проверим, что если последовательность $\varepsilon_{k}, k=1,2, \ldots$, стремится к нулю не слишком сильно, будем предполагать, что $\left|\varepsilon_{k}\right| \geqslant(\ln (k+1))^{-1 / 2}, k=1,2, \ldots$, то функция $u(r, \varphi)$ из (4) условию (3) удовлетворять не будет. Действительно, так как

$$
|\nabla u|^{2}=u_{r}^{2}+\frac{1}{r^{2}} u_{\varphi}^{2} \geqslant \frac{1}{r^{2}} u_{\varphi}^{2}
$$

а в силу (4)

$$
u_{\varphi}=-\left(1-r^{2}\right) \sum_{k=1}^{\infty} \varepsilon_{k} k^{3 / 2} r^{k} \sin k \varphi, \quad r<1
$$

то

$$
\begin{aligned}
\int_{|x|<1}|\nabla u|^{2}(1-|x|) d x & \geqslant \int_{|x|<1} \frac{1-|x|}{|x|^{2}} u_{\varphi}^{2} d x \\
& =\pi \int_{0}^{1} \frac{1-r}{r}\left(1-r^{2}\right)^{2} \sum_{k=1}^{\infty} \varepsilon_{k}^{2} k^{3} r^{2 k} d r \\
& \geqslant \frac{\pi}{4} \int_{0}^{1}\left(1-r^{2}\right)^{3} \sum_{k=1}^{\infty} \varepsilon_{k}^{2} k^{3} r^{2 k} d r^{2} \\
& \geqslant \sum_{k=1}^{\infty} \frac{k^{3}}{\ln (k+1)} \frac{\pi}{4} \int_{0}^{1}(1-\xi)^{3} \xi^{k} d \xi \\
& =\frac{\pi}{4} \sum_{k=1}^{\infty} \frac{k^{3}}{\ln (k+1)} B(4, k+1) \\
& =\frac{\pi \Gamma(4)}{4} \sum_{k=1}^{\infty} \frac{k^{3}}{(k+1) \cdots(k+4) \ln (k+1)}=\infty
\end{aligned}
$$


что и требовалось установить.

В работе получены критерии сушествования $L_{2}$-пределов на границе у решений уравнения (1). Для формулировки этих теорем нам понадобятся следующие обозначения.

На пространстве $L_{2}(0,2 \pi)$ введем семейство проекционных операторов. Оператор $P_{N}$ для любого целого $N \geqslant 1$ определим следующим образом. Для произвольной функции $f(\varphi) \in L_{2}(0,2 \pi)$, разложение которой в тригонометрический ряд Фурье имеет вид

$$
f(\varphi)=\frac{a_{0}}{2}+\sum_{k=1}^{\infty}\left(a_{k} \cos k \varphi+b_{k} \sin k \varphi\right),
$$

положим

$$
\left(P_{N} f\right)(\varphi)=\sum_{k=N+1}^{2 N}\left(a_{k} \cos k \varphi+b_{k} \sin k \varphi\right)=\sum_{(N, 2 N]}\left(a_{k} \cos k \varphi+b_{k} \sin k \varphi\right) .
$$

Обозначим через $\delta_{h}^{2}, h \geqslant 0$, оператор второй симметрической разности: для любой заданной на $\mathbb{R}_{1} 2 \pi$-периодической функции $f(\varphi)$ из $L_{2}(0,2 \pi)$

$$
\left(\delta_{h}^{2} f\right)(\varphi)=f(\varphi+h)-2 f(\varphi)+f(\varphi-h), \quad \varphi \in \mathbb{R}_{1}, \quad h \geqslant 0 .
$$

Гильбертово пространство, состоящее из всех определенных на $\mathbb{R}_{1}$ обобщенных $2 \pi$-периодических функций $f(\varphi)$, принадлежащих $W_{2}^{-1}(0,2 \pi)$, обозначим через $H^{-1}$; любую функцию $f(\varphi)$ из $H^{-1}$ можно представить в виде сходящегося в $H^{-1}$ ряда $(7)$; в качестве нормы в $H^{-1}$, эквивалентной обычной, можно взять выражение

$$
\|f\|_{H^{-1}}=\left(\frac{a_{0}^{2}}{2}+\sum_{k=1}^{\infty} \frac{a_{k}^{2}+b_{k}^{2}}{k^{2}}\right)^{1 / 2}
$$

Имеют место следующие утверждения.

Теорема 1. Пусть $u(x),|x|<1,-$ решение уравнения (1). Для существования $L_{2}$-предела на границе функции и $(x)$ необходимо и достаточно, чтобь эта функиия удовлетворяла условию (2), т.е. была $L_{2}$-ограниченной, и удовлетворяла условию

$$
\sup _{r<1}\left\|P_{N} u(r, \cdot)\right\|_{L_{2}} \rightarrow 0 \quad \text { npu } \quad N \rightarrow \infty .
$$

ТЕОРема 2. Пусть $и(x),|x|<1$, - решение уравнения (1). Для существования $L_{2}$-предела на границе функиии $и(x)$ необходимо и достаточно, чтобъ эта функиия удовлетворяла условию (2), т.е. бъла $L_{2}$-ограниченной, и удовлетворяла условию

$$
\sup _{r<1}\left\|\frac{\delta_{h}^{2} u(r, \cdot)}{h}\right\|_{H^{-1}} \rightarrow 0 \quad \text { npu } \quad h \rightarrow 0 .
$$

Из этих теорем вытекает еще один критерий.

ТеОрема 3. Пусть $u(x),|x|<1,-$ решение уравнения (1). Для существования $L_{2}$-предела на границе функиии и $(x)$ необходимо и достаточно, чтобь множество функиий $u(r, \varphi), r<1$, было компактным в $L_{2}(0,2 \pi)$.

Доказательства этих теорем основаны на ряде вспомогательных утверждений. В $\S 1$ эти вспомогательные утверждения формулируются и доказьваются, в $\S 2$ на основании доказанных в $\S 1$ лемм устанавливается справедливость теорем 1-3. 


\section{§ 1. Вспомогательные утверждения}

Важную роль в наших рассуждениях будут играть специальные полигармонические функции: при любом целом $k \geqslant 0$ и при любой гармонической функции $v(x) \oint у н к и и я ~ r^{2 k} v(x) \quad(k+1)$-гармонична. Это утверждение проще всего доказать индукцией по $k$. При $k=0$ оно очевидно; пусть оно установлено для всех $k<s$. Докажем его для $k=s$. Так как

$$
\Delta\left(r^{2 s} v\right)=\Delta\left(r^{2 s}\right) \cdot v+2\left(\nabla r^{2 s}, \nabla v\right)+r^{2 s} \Delta v=4 s^{2} r^{2(s-1)} v+4 s r^{2(s-1)}(\nabla v, x)
$$

и поскольку вместе с функцией $v(x)$ функция $(\nabla v, x)$ является гармонической, то по предположению индукции функция $\Delta\left(r^{2 s} v\right)$ является $s$-гармонической, и тем самым $r^{2 s} v$ является $(s+1)$-гармонической функцией. Из доказанного вытекает, что при любой гармонической функции $v(x)$ и любом целом $s \geqslant 0$ функция $\left(1-|x|^{2}\right)^{s} v(x)$ является $(s+1)$-гармонической, т.е. функции $\left(1-|x|^{2}\right)^{s} v(x)$ для всех целых $s \in[0, m-1]$ и всех гармонических $v(x)$ являются $m$-гармоническими функциями. Оказывается, что множество всех линейных комбинаций таких функций $\left(1-|x|^{2}\right)^{s} v(x), 0 \leqslant s \leqslant m-1$, совпадает с множеством всех $m$-гармонических функций. Имеет место следуюшее утверждение.

Лемма 1. Для любой т-гармонической в круге $\{|x|<1\}$ функиии и $(x)$ найдутся, причем единственным образом, гармонические в әтом круге функции $u_{0}(x), \ldots, u_{m-1}(x)$ maкие, что

$$
u(x)=u_{0}(x)+\left(1-|x|^{2}\right) u_{1}(x)+\cdots+\left(1-|x|^{2}\right)^{m-1} u_{m-1}(x), \quad|x|<1 .
$$

Конечно, представление $m$-гармонической функции в виде (10) не является новым: при $m=2$ оно имеется, например, в книге [2]. Однако, в известных мне его доказательствах используется дополнительная информация о поведении функции $u(x)$ при приближении к границе области. В связи с этим подчеркнем, что утверждение леммы 1 носит чисто локальньй характер: в ней не налагается никаких ограничений на поведение при приближении к границе входящих в формулу (10) функций. Именно в таком виде равенство (10) нами и будет использоваться.

ДокАЗАТЕЛЬСТво. Возьмем произвольное $R \in(0,1)$ и покажем, что функцией $u(x)$ однозначно определяются в круге $\{|x|<R\}$ такие гармонические функции $u_{0}^{\prime}(x), \ldots, u_{m-1}^{\prime}(x), u_{i}^{\prime}(x) \in C^{\infty}(|x| \leqslant R), i=0, \ldots, m-1$, что имеет место равенство

$$
u(x)=u_{0}^{\prime}(x)+\left(R^{2}-r^{2}\right) u_{1}^{\prime}(x)+\cdots+\left(R^{2}-r^{2}\right)^{m-1} u_{m-1}^{\prime}(x), \quad|x|<R .
$$

Предположим, что равенство (11) имеет место; докажем его единственность, при этом одновременно увидим, каким образом функции $u_{i}^{\prime}(x), i=0, \ldots, m-1$, определяются через $u(x)$. В силу (11) функция $u_{0}^{\prime}(x)$ обязана быть решением задачи Дирихле: $\Delta u_{0}^{\prime}=0,|x|<R,\left.u_{0}^{\prime}\right|_{|x|=R}=\left.u\right|_{|x|=R}$; таким образом, фууккция $u_{0}^{\prime}$ единственным образом определяется по функции $u$. Дифференцируя (11) по $r$ и полагая $r=R$, получим, что функция $u_{1}^{\prime}(x)$ обязана быть решением задачи Дирихле: $\Delta u_{1}^{\prime}=0,|x|<R,\left.u_{1}^{\prime}\right|_{|x|=R}=-\left.\frac{u}{2 R}\right|_{r=R}+\left.\frac{u_{0 r}^{\prime}}{2 R}\right|_{r=R}$; таким образом, функция $u_{1}^{\prime}(x)$ также однозначно определяется функцией $u(x)$ и т. д. Дифференцируя (11) 
по $r(m-1)$ раз и полагая $r=R$, получим, что функция $u_{m-1}^{\prime}(x)$ обязана быть решением задачи Дирихле:

$$
\begin{gathered}
\Delta u_{m-1}^{\prime}=0, \quad|x|<R, \\
\left.u_{m-1}^{\prime}\right|_{|x|=R}=\frac{(-1)^{m-1}}{(2 R)^{m-1}(m-1) !}\left[\left.u_{r^{m-1}}\right|_{r=R}-\left.u_{0 r^{m-1}}^{\prime}\right|_{r=R}+\cdots\right],
\end{gathered}
$$

где многоточием обозначены слагаемые, содержащие значения при $r=R$ производных по $r$ порядков, меньших $m-1$, уже определенных однозначно по $u(x)$ функций $u_{0}^{\prime}(x), \ldots, u_{m-2}^{\prime}(x)$. Таким образом, и функция $u_{m-1}^{\prime}$ однозначно определяется функцией $u$. Для доказательства существования разложения (11) достаточно подставить в $(11)$ в качестве функций $u_{0}^{\prime}(x), \ldots, u_{m-1}^{\prime}(x)$ только что найденные функции. При этом будет вьполняться тождество (11), так как и в правой, и в левой его частях стоят $m$-гармонические функции, удовлетворяющие одним и тем же условиям Дирихле для $m$-гармонического уравнения в круге $\{|x|<R\}$ : на окружности $\{|x|=R\}$ их производные по $r$ от нулевого до $(m-1)$-го порядков совпадают. Равенство (11) можно переписать в виде

$$
u(x)=u_{0}^{\prime \prime}(x)+r^{2} u_{1}^{\prime \prime}(x)+\cdots+r^{2(m-1)} u_{m-1}^{\prime \prime}(x), \quad|x|<R,
$$

где гармонические в $\{|x|<R\}$ и бесконечно дифференцируемые в $\{|x| \leqslant R\}$ функции $u_{0}^{\prime \prime}(x), \ldots, u_{m-1}^{\prime \prime}(x)$ однозначно определяются по функциям $u_{0}^{\prime}(x), \ldots, u_{m-1}^{\prime}(x)$ формулами

$$
\begin{aligned}
u_{m-1}^{\prime \prime} & =(-1)^{m-1} u_{m-1}^{\prime}, \\
& \cdots \\
u_{i}^{\prime \prime} & =(-1)^{m-1} C_{m-1}^{i} R^{2(m-1-i)} u_{m-1}^{\prime}+\cdots+(-1)^{i} C_{i}^{i} R^{0} u_{i}^{\prime}, \\
& \cdots \\
u_{0}^{\prime \prime} & =(-1)^{m-1} R^{2(m-1)} u_{m-1}^{\prime}+\cdots+(-1)^{0} R^{0} C_{0}^{0} u_{0}^{\prime} .
\end{aligned}
$$

Поскольку соответствие (13) между векторами $\left(u_{0}^{\prime}, \ldots, u_{m-1}^{\prime}\right)$ и $\left(u_{0}^{\prime \prime}, \ldots, u_{m-1}^{\prime \prime}\right)$, компоненты которых - гармонические функции, взаимно однозначное (с определителем матрицы, равным $\left.(-1)^{m(m-1) / 2}\right)$, то представление $(12) m$-гармонической функции в круге $\{|x| \leqslant R\}$ однозначно определено функцией $u(x)$.

Равенство (12) можно написать и в круге $\left\{|x|<R^{\prime}\right\}$ при любом $R^{\prime}$, $R<R^{\prime}<1$; при этом значения новых гармонических в круге $\left\{|x|<R^{\prime}\right\}$ функций $u_{0}^{\prime \prime}(x), \ldots, u_{m-1}^{\prime \prime}(x)$ обязаны в круге $\{|x|<R\}$ совпадать с соответствующими, найденными выше, значениями функций $u_{0}^{\prime \prime}(x), \ldots, u_{m-1}^{\prime \prime}(x)$. Это означает, что функции $u_{i}^{\prime \prime}(x), i=0, \ldots, m-1$, из формулы (12) на самом деле являются гармоническими функциями в круге $\left\{|x|<R^{\prime}\right\}$ и, тем самым, во всем круге $\{|x|<1\}$, и равенство (12) имеет место во всем круге $\{|x|<1\}$.

Функции $u_{0}(x), \ldots, u_{m-1}(x),|x|<1$, из разыскиваемого нами представления (10) находятся по функциям $u_{0}^{\prime \prime}(x), \ldots, u_{m-1}^{\prime \prime}(x),|x|<1$, как решение $\left(u_{0}^{\prime}, \ldots, u_{m-1}^{\prime}\right)$ системы $(13)$ при $R=1$ :

$$
\begin{gathered}
u_{0}^{\prime}=u_{0}, \ldots, u_{m-1}^{\prime}=u_{m-1}, \\
u_{i}(x)=(-1)^{m-1} C_{m-1}^{i} u_{m-1}^{\prime \prime}(x)+\cdots+(-1)^{i} C_{i}^{i} u_{i}^{\prime \prime}(x), \\
0 \leqslant i \leqslant m-1, \quad|x|<1
\end{gathered}
$$


Единственность представления $m$-гармонической функции в виде (10) вытекает из того, что определитель преобразования (13) при $R=1$ отличен от нуля. Лемма доказана.

Докажем несколько вспомогательных утверждений, касающихся отдельных слагаемых правой части равенства (10).

Пусть $v(x)=v(r, \varphi)$ - гармоническая в круге $\{r<1\}$ функция. Ее можно представить в виде

$$
v(r, \varphi)=\frac{a_{0}}{2}+\sum_{k=1}^{\infty}\left(a_{k} \cos k \varphi+b_{k} \sin k \varphi\right) r^{k}, \quad r<1,
$$

где ряд сходится равномерно вместе со всеми производньми на любом замкнутом подмножестве круга $\{r<1\}$.

Имеет место следующее элементарное утверждение, относящееся к нулевому (при $i=0)$ слагаемому правой части равенства (10).

ЛЕмма 2. Если гармоническая в круге $\{r<1\}$ функиия $v(r, \varphi)$ удовлетворяет неравенству $(2)$

$$
\sup _{r<1}\|v(r, \cdot)\|_{L_{2}}<\infty
$$

mo

$$
\sup _{r<1}\left\|P_{N} v(r, \cdot)\right\|_{L_{2}} \rightarrow 0 \quad \text { npu } \quad N \rightarrow \infty
$$

$u$

$$
\sup _{r<1}\left\|\frac{\delta_{h}^{2} v(r, \cdot)}{h}\right\|_{H^{-1}} \rightarrow 0 \quad n p u \quad h \rightarrow 0 .
$$

ДокАЗАТЕЛЬСтво. Условие $L_{2}$-ограниченности

$$
\frac{a_{0}^{2}}{2} \pi+\pi \sum_{k=1}^{\infty}\left(a_{k}^{2}+b_{k}^{2}\right) r^{2 k} \leqslant \mathscr{M}, \quad r<1
$$

где $\mathscr{M}$ - некоторая постоянная, влечет за собой сходимость ряда

$$
\sum_{k=1}^{\infty}\left(a_{k}^{2}+b_{k}^{2}\right)<\infty
$$

Следовательно, для всех $r<1$

$$
\left\|P_{N} v(r, \cdot)\right\|_{L_{2}}^{2}=\pi \sum_{(N, 2 N]}\left(a_{k}^{2}+b_{k}^{2}\right) r^{2 k} \leqslant \pi \sum_{(N, 2 N]}\left(a_{k}^{2}+b_{k}^{2}\right) \rightarrow 0 \text { при } N \rightarrow \infty
$$

и, тем самым, последовательность $\left\|P_{N} v(r, \cdot)\right\|_{L_{2}}, N=1,2, \ldots$, сходится к нулю равномерно по $r, r<1$, а значит, имеет место соотношение (15). Столь же просто доказывается и соотношение (16). Поскольку

$$
\delta_{h}^{2} v(r, \varphi)=-2 \sum_{k=1}^{\infty}\left(a_{k} \cos k \varphi+b_{k} \sin k \varphi\right) r^{k}(1-\cos k h)
$$


то для всех $h>0$ и $r<1$

$$
\begin{aligned}
\left\|\frac{\delta_{h}^{2} v(r, \cdot)}{h}\right\|_{H^{-1}}^{2} & =4 \sum_{k=1}^{\infty}\left(a_{k}^{2}+b_{k}^{2}\right) r^{2 k} \frac{(1-\cos k h)^{2}}{k^{2} h^{2}} \\
& \leqslant 4 \sum_{k=1}^{\infty}\left(a_{k}^{2}+b_{k}^{2}\right) \frac{(1-\cos k h)^{2}}{k^{2} h^{2}} \rightarrow 0 \text { при } h \rightarrow 0,
\end{aligned}
$$

так как при каждом $k, k=1,2, \ldots, \frac{(1-\cos k h)^{2}}{k^{2} h^{2}} \rightarrow 0$ при $h \rightarrow 0$, а последний ряд сходится в силу неравенства $0 \leqslant \frac{(1-\cos k h)^{2}}{k^{2} h^{2}} \leqslant 1$ равномерно по $h, h>0$. Лемма доказана.

Обратимся к оставшимся слагаемым, $i=1, \ldots, m-1$, правой части равенства (10).

ЛЕмма 3. Пусть (иелое) число $p>0, a v(x)$ - гармоническая в круге $\{|x|<1\}$ функиия, разложение в ряд Фурье которой имеет вид (14). Для того чтоби выполнялось равенство

$$
\left\|\left(1-r^{2}\right)^{p} v(r, \cdot)\right\|_{L_{2}}=O(1) \quad n p u \quad r \rightarrow 1
$$

необходимо и достаточно, чтобъ имело место соотношение

$$
\sum_{(N, 2 N]} \frac{a_{k}^{2}+b_{k}^{2}}{k^{2 p}}=O(1) \quad n p u \quad N \rightarrow \infty
$$

Для того чтобы выполнялось равенство

$$
\left\|\left(1-r^{2}\right)^{p} v(r, \cdot)\right\|_{L_{2}}=o(1) \quad n p u \quad r \rightarrow 1
$$

необходимо и достаточно, чтобъ имело место соотношение

$$
\sum_{(N, 2 N]} \frac{a_{k}^{2}+b_{k}^{2}}{k^{2 p}}=o(1) \quad \text { npu } \quad N \rightarrow \infty
$$

ДокАЗАТЕльство. Вначале покажем, что из (17) и (19) вытекают, соответственно, (18) и (20). Так как

$$
\left\|\left(1-r^{2}\right)^{p} v(r, \cdot)\right\|_{L_{2}}^{2}=\pi\left(1-r^{2}\right)^{2 p}\left(\frac{a_{0}^{2}}{2}+\sum_{k=1}^{\infty}\left(a_{k}^{2}+b_{k}^{2}\right) r^{2 k}\right),
$$

то (17) можно переписать в виде неравенства

$$
\left(1-r^{2}\right)^{2 p} \sum_{k=1}^{\infty}\left(a_{k}^{2}+b_{k}^{2}\right) r^{2 k} \leqslant \mathscr{M}, \quad r \in(0,1)
$$


при некоторой постоянной $\mathscr{M}>0$ или, что то же самое (после замены переменной $\left.1-r^{2}=x, x \in(0,1)\right),-$ в виде неравенства

$$
\sum_{k=1}^{\infty}\left(a_{k}^{2}+b_{k}^{2}\right) x^{2 p}(1-x)^{k} \leqslant \mathscr{M}, \quad x \in(0,1) .
$$

Заметим, что в случае выполнения соотношения (19) наряду с $\left(17^{\prime}\right)$ имеет место и аналогичное неравенство с $\mathscr{M}$ сколь угодно малым, выполняющееся уже, вообще говоря, не для всех $x \in(0,1)$, а лишш для всех $x$ из $(0,1)$, достаточно близких к нулю $(x \rightarrow 0$ при $r \rightarrow 1)$.

Из $\left(17^{\prime}\right)$ имеем неравенство

$$
\begin{aligned}
\mathscr{M} & \geqslant \sum_{k=1}^{\infty} \frac{a_{k}^{2}+b_{k}^{2}}{k^{2 p}}(1-x)^{k}(k x)^{2 p} \\
& \geqslant \sum_{k \in\left(\frac{1}{x}, \frac{2}{x}\right]} \frac{a_{k}^{2}+b_{k}^{2}}{k^{2 p}}(1-x)^{k}(k x)^{2 p} \\
& \geqslant \sum_{\left(\frac{1}{x}, \frac{2}{x}\right]} \frac{a_{k}^{2}+b_{k}^{2}}{k^{2 p}}\left(1-\frac{2}{k}\right)^{k} \\
& \geqslant \frac{e^{-2}}{2} \sum_{\left(\frac{1}{x}, \frac{2}{x}\right]} \frac{a_{k}^{2}+b_{k}^{2}}{k^{2 p}}
\end{aligned}
$$

из которого при достаточно малых $x$ или достаточно больших $N=1 / x$ получаем неравенство

$$
\sum_{(N, 2 N]} \frac{a_{k}^{2}+b_{k}^{2}}{k^{2 p}} \leqslant 2 \mathscr{M} e^{-2},
$$

совпадаюшее с соотношением (18), если имеет место (17), и в силу сделанного выше замечания - с соотношением (20), если имеет место (19).

Докажем теперь, что из (18) и (20) вытекают, соответственно, соотношения (17) и (19).

Левую часть $\left(17^{\prime}\right)$ с помощью преобразования Абеля представим в виде

$$
\begin{aligned}
\sum_{k=1}^{\infty} & \frac{a_{k}^{2}+b_{k}^{2}}{k^{2 p}}(1-x)^{k}(k x)^{2 p} \\
& =\sum_{k=1}^{\infty} k \frac{a_{k}^{2}+b_{k}^{2}}{k^{2 p}} \frac{(k x)^{2 p}(1-x)^{k}}{k} \\
& =\sum_{k=1}^{\infty}(\mathscr{F}(k)-\mathscr{F}(k-1)) \frac{(k x)^{2 p}(1-x)^{k}}{k} \\
& =\sum_{k=1}^{\infty} \mathscr{F}(k)\left[\frac{(k x)^{2 p}(1-x)^{k}}{k}-\frac{((k+1) x)^{2 p}(1-x)^{k+1}}{k+1}\right] \\
& =\sum_{k=1}^{\infty} \frac{1}{k} \mathscr{F}(k) \psi_{k}(x),
\end{aligned}
$$


где

$$
\mathscr{F}(k)=\sum_{i=1}^{k} i \frac{a_{i}^{2}+b_{i}^{2}}{i^{2 p}}, \quad k \geqslant 1, \quad \mathscr{F}(0)=0,
$$

a

$$
\begin{aligned}
\psi_{k}(x) & =k\left(\frac{(k x)^{2 p}(1-x)^{k}}{k}-\frac{((k+1) x)^{2 p}(1-x)^{k+1}}{k+1}\right) \\
& =k^{2 p} x^{2 p}(1-x)^{k}-k(k+1)^{2 p-1} x^{2 p}(1-x)^{k+1} \\
& =x^{2 p}(1-x)^{k}\left(k^{2 p}-k(k+1)^{2 p-1}\right)+x^{2 p+1} k(k+1)^{2 p-1}(1-x)^{k} \\
& =-x^{2 p}(1-x)^{k} \sum_{j=0}^{2 p-2} C_{2 p-1}^{j} k^{j+1}+x^{2 p+1}(1-x)^{k} \sum_{j=0}^{2 p-1} C_{2 p-1}^{j} k^{j+1}
\end{aligned}
$$

Следовательно,

$$
\left|\psi_{k}(x)\right| \leqslant x^{2 p}(1-x)^{k} \sum_{j=0}^{2 p-2} C_{2 p-1}^{j} k^{j+1}+x^{2 p+1}(1-x)^{k} \sum_{j=0}^{2 p-1} C_{2 p-1}^{j} k^{j+1}
$$

Легко видеть, что для любого (целого) $\alpha \geqslant 0$ существует постоянная $C_{\alpha}>0$ такая, что

$$
\sum_{k=1}^{\infty} k^{\alpha}(1-x)^{k} \leqslant \frac{C_{\alpha}}{x^{\alpha+1}} \text { для всех } x \in(0,1) .
$$

При $\alpha=0$ это неравенство вытекает из разложения

$$
\frac{1}{x}=\sum_{k=0}^{\infty}(1-x)^{k}, \quad 0<x<2
$$

Для доказательства его справедливости при любом (целом) $\alpha>0$ продифференцируем ряд (25) $\alpha$ раз. В результате получим неравенство

$$
\begin{aligned}
\frac{\alpha !}{x^{\alpha+1}} & =\sum_{k=0}^{\infty} k(k-1) \cdots(k-\alpha+1)(1-x)^{k-\alpha} \\
& =\sum_{k=1}^{\infty}(k+\alpha-1) \cdots k(1-x)^{k-1} \\
& =\frac{1}{1-x} \sum_{k=1}^{\infty}(k+\alpha-1) \cdots k(1-x)^{k} \\
& \geqslant \frac{1}{1-x} \sum_{k=1}^{\infty} k^{\alpha}(1-x)^{k}, \quad 0<x<2
\end{aligned}
$$

из которого (24) немедленно следует. 
Из (23) и (24) для всех $x \in(0,1)$ имеем неравенство

$$
\begin{aligned}
\sum_{k=1}^{\infty}\left|\psi_{k}(x)\right| \leqslant & x^{2 p} \sum_{j=0}^{2 p-2} C_{2 p-1}^{j} \sum_{k=1}^{\infty} k^{j+1}(1-x)^{k} \\
& +x^{2 p-1} \sum_{j=0}^{2 p-1} C_{2 p-1}^{j} \sum_{k=1}^{\infty} k^{j+1}(1-x)^{k} \\
\leqslant & \sum_{j=0}^{2 p-2} C_{2 p-1}^{j} C_{j+1} x^{2 p-j-2} \\
& +\sum_{j=0}^{2 p-1} C_{2 p-1}^{j} C_{j+1} x^{2 p+1-j-2} \leqslant \mathscr{M}_{1}, \quad x \in(0,1),
\end{aligned}
$$

где $\mathscr{M}_{1}$ - некоторая постоянная.

Имеет место следуюшая

Лемма 4. Пусть $\gamma_{1}, \gamma_{2}, \ldots$ - последовательность неотрицательных чисел. Ecлu

$$
\sum_{(N, 2 N]} \gamma_{k}=O(1) \quad n p u \quad N \rightarrow \infty
$$

mo

$$
\frac{1}{N} \sum_{k=1}^{N} k \gamma_{k}=O(1) \quad \text { npu } \quad N \rightarrow \infty
$$

Eсли

$$
\sum_{(N, 2 N]} \gamma_{k}=o(1) \quad \text { npu } \quad N \rightarrow \infty
$$

mo

$$
\frac{1}{N} \sum_{k=1}^{N} k \gamma_{k}=o(1) \quad n p u \quad N \rightarrow \infty .
$$

Продолжим доказательство леммы 3 , воспользовавшись леммой 4; лемму 4 докажем позднее. Если положить $\gamma_{k}=\left(a_{k}^{2}+b_{k}^{2}\right) / k^{2 p}, k \geqslant 1$, то в силу (18) и (20) имеем, соответственно, равенства (27) и (29). Следовательно, согласно лемме 4 числовая последовательность $\mathscr{F}(k), k=0,1, \ldots$, определенная равенствами $(22)$, обладает при некоторой постоянной $\mathscr{M}_{2}>0$ свойством

$$
0 \leqslant \frac{\mathscr{F}(k)}{k} \leqslant \mathscr{M}_{2} \text { для всех } k \geqslant 1,
$$

если выполнено соотношение (18), а если вьполнено соотношение (20), то по любому $\varepsilon>0$ можно найти такое $k_{0}=k_{0}(\varepsilon)>0$, что

$$
0 \leqslant \frac{\mathscr{F}(k)}{k} \leqslant \varepsilon \text { для всех } k \geqslant k_{0},
$$

неравенство (31) и в этом случае, конечно, тоже выполняется. 
Продолжим равенство (21). В случае, если имеет место (18), в силу (31) и (26) имеем для всех $x \in(0,1)$

$$
\sum_{k=1}^{\infty}\left(a_{k}^{2}+b_{k}^{2}\right) x^{2 p}(1-x)^{k} \leqslant \mathscr{M}_{2} \sum_{k=1}^{\infty}\left|\psi_{k}(x)\right| \leqslant \mathscr{M}_{2} \mathscr{M}_{1},
$$

т.е. имеет место $\left(17^{\prime}\right)$, а значит, и (17) $\left(x=1-r^{2}\right)$. В случае, если имеет место $(20)$, в силу $(31),(32)$ и $(26)$ для всех $x \in(0,1)$

$$
\begin{aligned}
& \sum_{k=1}^{\infty}\left(a_{k}^{2}+b_{k}^{2}\right)\left(1-r^{2}\right)^{2 p} r^{2 k} \\
& \quad=\sum_{k=1}^{\infty}\left(a_{k}^{2}+b_{k}^{2}\right) x^{2 p}(1-x)^{k} \\
& \quad \leqslant \sum_{k=1}^{k_{0}} \frac{\mathscr{F}(k)}{k}\left|\psi_{k}(x)\right|+\sum_{k=k_{0}+1}^{\infty} \frac{\mathscr{F}(k)}{k}\left|\psi_{k}(x)\right| \\
& \quad \leqslant \max _{1 \leqslant k \leqslant k_{0}}\left|\psi_{k}(x)\right| \sum_{k=1}^{k_{0}} \frac{\mathscr{F}(k)}{k}+\varepsilon \sum_{k=1}^{\infty}\left|\psi_{k}(x)\right| \\
& \quad \leqslant \mathscr{M}_{2} k_{0} \max _{1 \leqslant k \leqslant k_{0}}\left|\psi_{k}(x)\right|+\varepsilon \mathscr{M}_{1}
\end{aligned}
$$

и, следовательно, для всех достаточно малых $x$, т.е. для всех $r$, достаточно близких к единице,

$$
\sum_{k=1}^{\infty}\left(a_{k}^{2}+b_{k}^{2}\right)\left(1-r^{2}\right)^{2 p} r^{2 k} \leqslant \varepsilon\left(\mathscr{M}_{2} k_{0}+\mathscr{M}_{1}\right),
$$

поскольку $\max _{1 \leqslant k \leqslant k_{0}}\left|\psi_{k}(x)\right| \rightarrow 0$ при $x \rightarrow 0$. Что и требовалось установить. Лемма 3 доказана.

ДоКАЗАТЕЛЬСТВо ЛЕМмы 4. Положим для любого целого $N \geqslant 1$

$$
\mathscr{F}(N)=\sum_{k=1}^{N} k \gamma_{k} .
$$

Нам нужно доказать, что

$$
\frac{1}{N} \mathscr{F}(N)=O(1) \text { при } N \rightarrow \infty,
$$

если имеет место (27), и

$$
\frac{1}{N} \mathscr{F}(N)=o(1) \text { при } N \rightarrow \infty,
$$

если имеет место (29).

Для любого целого $n \geqslant 1$ определим функцию $G(n)$ :

$$
G(n)=\sum_{\left(2^{n-1}, 2^{n}\right]} k \gamma_{k}=\mathscr{F}\left(2^{n}\right)-\mathscr{F}\left(2^{n-1}\right) .
$$


Так как $0 \leqslant G(n) \leqslant 2^{n} \sum_{\left(2^{n-1}, 2^{n}\right]} \gamma_{k}$, то в случае $(27)$

$$
G(n) \leqslant \mathscr{M} \cdot 2^{n} \text { для всех } n \geqslant 1
$$

где $\mathscr{M}>0$ - некоторая постоянная, а в случае (29) по любому $\varepsilon>0$ можно найти такое $n_{0}$, что

$$
G(n) \leqslant \varepsilon \cdot 2^{n} \text { для всех } n \geqslant n_{0},
$$

при этом, конечно, имеет место и неравенство (36).

Для любого целого $n \geqslant 1$ в силу (35) имеем равенство

$$
\begin{aligned}
\mathscr{F}\left(2^{n}\right) & =\mathscr{F}\left(2^{n-1}\right)+G(n)=\mathscr{F}\left(2^{n-2}\right)+G(n)+G(n-1) \\
& =\cdots=\mathscr{F}(1)+G(n)+G(n-1)+\cdots+G(2) .
\end{aligned}
$$

Возьмем произвольное целое $N>1$ и обозначим через $n$ (единственное) целое число, удовлетворяющее неравенствам

$$
2^{n-1}<N \leqslant 2^{n}, \quad \text { т.е. } \frac{\ln N}{\ln 2} \leqslant n<\frac{\ln N}{\ln 2}+1 .
$$

Если имеет место равенство (27), а значит, и неравенство (35), то из (38) имеем

$$
\begin{aligned}
\frac{\mathscr{F}(N)}{N} & \leqslant \frac{\mathscr{F}\left(2^{n}\right)}{2^{n-1}} \leqslant \frac{\mathscr{F}(1)+G(2)+\cdots+G(n)}{2^{n-1}} \\
& \leqslant \frac{\mathscr{F}(1)}{2^{n-1}}+\mathscr{M} \frac{2^{1}+\cdots+2^{n}}{2^{n-1}} \leqslant \frac{\mathscr{F}(1)}{2^{n-1}}+4 \mathscr{M}
\end{aligned}
$$

откуда вытекает (33). Если имеет место равенство (29), то при произвольно взятом $\varepsilon>0$ имеет место неравенство (37), из которого в силу (38) имеем

$$
\begin{aligned}
\frac{\mathscr{F}(N)}{N} & \leqslant \frac{\mathscr{F}(1)+G(2)+\cdots+G\left(n_{0}-1\right)+G\left(n_{0}\right)+\cdots+G(n)}{2^{n-1}} \\
& \leqslant \frac{\mathscr{F}(1)+G(2)+\cdots+G\left(n_{0}-1\right)}{2^{n-1}}+\varepsilon \frac{2^{n_{0}}+\cdots+2^{n}}{2^{n-1}} \\
& \leqslant \frac{\mathscr{F}(1)+G(2)+\cdots+G\left(n_{0}-1\right)}{2^{n-1}}+4 \varepsilon,
\end{aligned}
$$

откуда при достаточно большом $N$ (а значит, и $n) \mathscr{F}(N) / N \leqslant 5 \varepsilon$. Что и требовалось установить. Лемма 4 доказана.

Кроме леммы 4 в дальнейшем нам понадобится следующая элементарная

Лемма 5. Пусть $\gamma_{1}, \gamma_{2}, \ldots$ - последовательность неотрицательных чисел u nycmb

$$
\sum_{(N, 2 N]} \gamma_{k}=O(1) \quad \text { npu } \quad N \rightarrow \infty .
$$

Тогда при любом $\sigma>0$ сходится ряд

$$
\sum_{k=1}^{\infty} \frac{\gamma_{k}}{k^{\sigma}}<\infty
$$


ДОКАЗАТЕЛЬСТво. В силу леммы 4

$$
\sum_{k=1}^{N} k \gamma_{k} \leqslant \mathscr{M} N \text { для всех } N \geqslant 1,
$$

где $\mathscr{M}$ - некоторая положительная постоянная. Введем обозначение:

$$
\mathscr{F}(k)=\sum_{i=1}^{k} i \gamma_{i}, \quad k \geqslant 1, \quad \mathscr{F}(0)=0 .
$$

С помощью преобразования Абеля имеем

$$
\begin{aligned}
\sum_{k=1}^{\infty} \frac{\gamma_{k}}{k^{\sigma}} & =\sum_{k=1}^{\infty} \frac{\gamma_{k} k}{k^{\sigma+1}}=\sum_{k=1}^{\infty}(\mathscr{F}(k)-\mathscr{F}(k-1)) \frac{1}{k^{\sigma+1}} \\
& =\sum_{k=1}^{\infty} \mathscr{F}(k)\left(\frac{1}{k^{\sigma+1}}-\frac{1}{(k+1)^{\sigma+1}}\right) \\
& =\sum_{k=1}^{\infty} \frac{\mathscr{F}(k)}{k^{\sigma+1}}\left(1-\left(1+\frac{1}{k}\right)^{-\sigma-1}\right) .
\end{aligned}
$$

Так как

$$
\left(1-\left(1+\frac{1}{k}\right)^{-\sigma-1}\right) / \frac{1}{k} \rightarrow \sigma+1 \text { при } k \rightarrow \infty,
$$

то для достаточно больших $k$

$$
\mathscr{F}(k) \frac{1}{k^{\sigma+1}}\left(1-\left(1+\frac{1}{k}\right)^{-\sigma-1}\right) \leqslant \frac{2(\sigma+1) \mathscr{F}(k)}{k^{\sigma+2}} \leqslant \frac{2 \mathscr{M}(\sigma+1)}{k^{\sigma+1}},
$$

т.е. исследуемый ряд сходится. Лемма доказана.

Лемма 6. Пусть (целое) $p>0, a v(r, \varphi)$ - гармоническая в круге $\{r<1\}$ функция. Следующие три утверждения әквивалентны.

I. $\left\|\left(1-r^{2}\right)^{p} v(r, \cdot)\right\|_{L_{2}} \rightarrow 0$ npu $r \rightarrow 1$.

II. $\sup _{r<1}\left\|P_{N}\left(1-r^{2}\right)^{p} v(r, \cdot)\right\|_{L_{2}} \rightarrow 0$ nрu $N \rightarrow \infty$.

III. $\sup _{r<1}\left\|\frac{\delta_{h}^{2}\left(1-r^{2}\right)^{p} v(r, \cdot)}{h}\right\|_{H^{-1}} \rightarrow 0$ npu $h \rightarrow 0$.

Пусть разложение в ряд Фурье функции $v(r, \varphi)$ задается формулой (14). В силу леммы 3 утверждение I эквивалентно следуюшему свойству коэффициентов Фурье функции $v(r, \varphi)$ :

$$
\sum_{(N, 2 N]} \frac{a_{k}^{2}+b_{k}^{2}}{k^{2 p}} \rightarrow 0 \text { при } N \rightarrow \infty .
$$

Свойство II в терминах коэффициентов Фурье функции $v(r, \varphi)$ имеет вид

$$
\sup _{r<1} \sum_{(N, 2 N]}\left(1-r^{2}\right)^{2 p}\left(a_{k}^{2}+b_{k}^{2}\right) r^{2 k} \rightarrow 0 \text { при } N \rightarrow \infty,
$$


а свойство III - имеет вид

$$
\sup _{r<1} \sum_{k=1}^{\infty} \frac{a_{k}^{2}+b_{k}^{2}}{k^{2} h^{2}} r^{2 k}\left(1-r^{2}\right)^{2 p}(1-\cos k h)^{2} \rightarrow 0 \text { при } h \rightarrow 0 .
$$

Для доказательства леммы нам следует установить эквивалентность соотношений $(39),(40)$ и (41).

Пусть справедливо соотношение (39). Докажем, что имеет место (40) и имеет место (41). Действительно, функция $\left(1-r^{2}\right)^{2 p} r^{2 k}, 0 \leqslant r \leqslant 1$, достигает своего максимального значения при $r=\left(\frac{k}{2 p+k}\right)^{1 / 2}$, и это максимальное значение равно $\frac{(2 p)^{2 p}}{(k+2 p)^{2 p}}\left(1+\frac{2 p}{k}\right)^{-k}$. Поэтому для всех $r \in(0,1)$ и всех достаточно больших $N$ имеет место неравенство

$$
\begin{aligned}
& \sum_{(N, 2 N]}\left(a_{k}^{2}+b_{k}^{2}\right)\left(1-r^{2}\right)^{2 p} r^{2 k} \leqslant \\
& \leqslant(2 p)^{2 p} \sum_{(N, 2 N]} \frac{a_{k}^{2}+b_{k}^{2}}{(k+2 p)^{2 p}}\left(1+\frac{2 p}{k}\right)^{-k} \\
& \leqslant 2(2 p)^{2 p} e^{-2 p} \sum_{(N, 2 N]} \frac{a_{k}^{2}+b_{k}^{2}}{k^{2 p}}
\end{aligned}
$$

показывающее, что из (39) вытекает (40).

Докажем теперь, что из (39) следует (41). Для всех $h>0$ и всех $r_{0} \in(0,1)$

$$
\begin{aligned}
\sup _{r<1} \sum_{k=1}^{\infty} \frac{a_{k}^{2}+b_{k}^{2}}{k^{2} h^{2}} r^{2 k}\left(1-r^{2}\right)^{2 p}(1-\cos k h)^{2} \\
\leqslant \sup _{r \leqslant r_{0}} \sum_{k=1}^{\infty} \frac{a_{k}^{2}+b_{k}^{2}}{k^{2} h^{2}} r^{2 k}\left(1-r^{2}\right)^{2 p}(1-\cos k h)^{2} \\
\quad+\sup _{r_{0} \leqslant r<1} \sum_{k=1}^{\infty} \frac{a_{k}^{2}+b_{k}^{2}}{k^{2} h^{2}} r^{2 k}\left(1-r^{2}\right)^{2 p}(1-\cos k h)^{2}
\end{aligned}
$$

Так как для всех $h>0$ и $k \geqslant 1 \frac{(1-\cos k h)^{2}}{k^{2} h^{2}}=4 \frac{\sin ^{4} \frac{k h}{2}}{k^{2} h^{2}} \leqslant 1$, то

$$
\begin{aligned}
& \sup _{r_{0} \leqslant r<1} \sum_{k=1}^{\infty} \frac{a_{k}^{2}+b_{k}^{2}}{k^{2} h^{2}} r^{2 k}\left(1-r^{2}\right)^{2 p}(1-\cos k h)^{2} \\
& \quad \leqslant \sup _{r_{0} \leqslant r<1} \sum_{k=1}^{\infty}\left(a_{k}^{2}+b_{k}^{2}\right) r^{2 k}\left(1-r^{2}\right)^{2 p} \\
& \quad=\frac{1}{\pi} \sup _{r_{0} \leqslant r<1}\left\|\left(1-r^{2}\right)^{p} v(r, \cdot)\right\|_{L_{2}}^{2},
\end{aligned}
$$


и, следовательно, в силу I по произвольному $\varepsilon>0$ найдется такое $r_{0}=r_{0}(\varepsilon)<1$, что

$$
\sup _{r_{0} \leqslant r<1} \sum_{k=1}^{\infty} \frac{a_{k}^{2}+b_{k}^{2}}{k^{2} h^{2}} r^{2 k}\left(1-r^{2}\right)^{2 p}(1-\cos k h)^{2} \leqslant \varepsilon \quad \text { для всех } h>0 .
$$

Первое слагаемое правой части (42) при выбранном $r_{0}<1$ оценивается так:

$$
\begin{aligned}
& \sup _{r \leqslant r_{0}} \sum_{k=1}^{\infty} \frac{a_{k}^{2}+b_{k}^{2}}{k^{2} h^{2}} r^{2 k}\left(1-r^{2}\right)^{2 p}(1-\cos k h)^{2} \\
& \quad \leqslant 4 \sum_{k=1}^{\infty}\left(a_{k}^{2}+b_{k}^{2}\right) r_{0}^{2 k} \frac{\sin ^{4} \frac{k h}{2}}{k^{2} h^{2}} \\
& \quad \leqslant \sum_{k=1}^{\infty}\left(a_{k}^{2}+b_{k}^{2}\right) r_{0}^{2 k} \sin ^{2} \frac{k h}{2} .
\end{aligned}
$$

Последний ряд сходится равномерно по $h>0$, поскольку в силу (39) и леммы 5 $a_{k}^{2}+b_{k}^{2} \leqslant$ const $k^{2 p+1}$ для всех $k \geqslant 1$, следовательно, его сумма при достаточно малом $h,|h| \leqslant h_{0}=h_{0}(\varepsilon)$, может быть сделана меньшей, чем $\varepsilon$. Тогда из $(42),(43)$ и $(44)$ для $|h| \leqslant h_{0}$ имеем

$$
\sup _{r<1} \sum_{k=1}^{\infty} \frac{a_{k}^{2}+b_{k}^{2}}{k^{2} h^{2}} r^{2 k}\left(1-r^{2}\right)^{2 p}(1-\cos k h)^{2} \leqslant 2 \varepsilon .
$$

Что и требовалось установить.

Покажем теперь, что свойство (39) вытекает как из (40), так и из (41).

Пусть имеет место соотношение (40). Тогда по любому $\varepsilon>0$ найдется такое $N_{0}=N_{0}(\varepsilon)$, что

$$
\sum_{(N, 2 N]}\left(1-r^{2}\right)^{2 p} r^{2 k}\left(a_{k}^{2}+b_{k}^{2}\right) \leqslant \varepsilon \text { для всех } r<1 \text { и всех } N \geqslant N_{0} .
$$

Подставляя в это неравенство $r=\left(1-\frac{1}{N}\right)^{1 / 2}$, получим

$$
\begin{aligned}
\varepsilon & \geqslant \sum_{(N, 2 N]}\left(a_{k}^{2}+b_{k}^{2}\right) \frac{1}{N^{2 p}}\left(1-\frac{1}{N}\right)^{k} \\
& \geqslant \sum_{(N, 2 N]} \frac{a_{k}^{2}+b_{k}^{2}}{k^{2 p}}\left(1-\frac{2}{k}\right)^{k} \geqslant \frac{e^{-2}}{2} \sum_{(N, 2 N]} \frac{a_{k}^{2}+b_{k}^{2}}{k^{2 p}},
\end{aligned}
$$

если только число $N_{0}$ достаточно велико. Соотношение (39) установлено.

Пусть, наконец, справедливо (41). Тогда по произвольному $\varepsilon>0$ найдется такое $h_{0}=h_{0}(\varepsilon)>0$, что

$$
\sum_{k=1}^{\infty} \frac{a_{k}^{2}+b_{k}^{2}}{h^{2} k^{2}} r^{2 k}\left(1-r^{2}\right)^{2 p}(1-\cos k h)^{2} \leqslant \varepsilon \quad \text { для всех } r<1 \text { и всех } h \leqslant h_{0} .
$$


Поскольку

$$
\frac{(1-\cos k h)^{2}}{h^{2} k^{2}}=\frac{\sin ^{4} \frac{k h}{2}}{\frac{h^{2} k^{2}}{4}} \geqslant \gamma=\min \left\{\sin ^{4} 1, \frac{\sin ^{4} 2}{4}\right\}>0
$$

для всех $h \leqslant h_{0}, k \geqslant 1$, для которых $\frac{1}{2} k h \in(1,2]$, то из неравенства (45) имеем неравенство, справедливое для всех $r<1, h \leqslant h_{0}$,

$$
\begin{aligned}
\varepsilon & \geqslant \sum_{\frac{2}{h} \leqslant k \leqslant \frac{4}{h}}\left(a_{k}^{2}+b_{k}^{2}\right) r^{2 k}\left(1-r^{2}\right)^{2 p} \frac{(1-\cos k h)^{2}}{(k h)^{2}} \\
& \geqslant \gamma \sum_{\frac{2}{h}<k \leqslant 2 \cdot \frac{2}{h}}\left(a_{k}^{2}+b_{k}^{2}\right) r^{2 k}\left(1-r^{2}\right)^{2 p}
\end{aligned}
$$

после подстановки в которое $r=(1-h)^{1 / 2}$ (считаем, что $\left.h_{0}<1\right)$, получаем неравенство

$$
\begin{aligned}
\varepsilon & \geqslant \gamma \sum_{\left(\frac{2}{h}, 2 \cdot \frac{2}{h}\right]}\left(a_{k}^{2}+b_{k}^{2}\right)(1-h)^{k} h^{2 p} \\
& \geqslant \gamma \sum_{\left(\frac{2}{h}, 2 \cdot \frac{2}{h}\right]}\left(a_{k}^{2}+b_{k}^{2}\right)\left(1-\frac{4}{k}\right)^{k}\left(\frac{2}{k}\right)^{2 p} \\
& \geqslant \frac{\gamma}{2} e^{-4} 2^{2 p} \sum_{\left(\frac{2}{h}, 2 \cdot \frac{2}{h}\right]} \frac{a_{k}^{2}+b_{k}^{2}}{k^{2 p}}
\end{aligned}
$$

совпадающее при $2 / h=N$ с неравенством (39). Лемма доказана.

ЛЕмма 7. т-гармоническая функиия $и(x)$ L-ограничена тогда и только тогда, когда $L_{2}$-ограничено кажсдое из слагаемых $\left(1-|x|^{2}\right)^{i} u_{i}(x), i=0, \ldots, m-1$, равенства (10), т.е. свойства

1) $\sup _{r<1}\|u(r, \cdot)\|_{L_{2}}<\infty$

$u$

2) $\sup _{r<1}\left\|\left(1-r^{2}\right)^{i} u_{i}(r, \cdot)\right\|_{L_{2}}<\infty, \quad i=0, \ldots, m-1$,

әквивалентны.

Ясно, что в доказательстве нуждается лишь утверждение, что из 1) следует 2), поскольку обратное утверждение очевидно.

Каждую гармоническую функцию $u_{i}(x)$ в (10) представим в виде

$$
u_{i}(r, \varphi)=\frac{a_{0 i}}{2}+\sum_{k=1}^{\infty}\left(a_{k i} \cos k \varphi+b_{k i} \sin k \varphi\right) r^{k}, \quad r<1, \quad i=0, \ldots, m-1
$$


Тогда при всех $r<1$

$$
\begin{aligned}
\|u(r, \cdot)\|_{L_{2}}^{2}= & \frac{\pi}{2}\left(\sum_{i=0}^{m-1} a_{0 i}\left(1-r^{2}\right)^{i}\right)^{2}+\pi \sum_{k=1}^{\infty}\left(\sum_{i=0}^{m-1} a_{k i}\left(1-r^{2}\right)^{i} r^{k}\right)^{2} \\
& +\pi \sum_{k=1}^{\infty}\left(\sum_{i=0}^{m-1} b_{k i}\left(1-r^{2}\right)^{i} r^{k}\right)^{2}, \quad r<1
\end{aligned}
$$

и из неравенства (46) вытекает неравенство

$$
\sum_{k=1}^{\infty}\left(\sum_{i=0}^{m-1} a_{k i}\left(1-r^{2}\right)^{i} r^{k}\right)^{2} \leqslant \mathscr{M}, \quad r<1
$$

и неравенство

$$
\sum_{k=1}^{\infty}\left(\sum_{i=0}^{m-1} b_{k i}\left(1-r^{2}\right)^{i} r^{k}\right)^{2} \leqslant \mathscr{M}, \quad r<1,
$$

где $\mathscr{M}$ - некоторая положительная постоянная. Неравенства в (47), в свою очередь, эквивалентны совокупности следующих неравенств для коэффициентов $a_{k i}$ :

$$
\sum_{k=1}^{\infty}\left(a_{k i}\left(1-r^{2}\right)^{i} r^{k}\right)^{2} \leqslant \mathscr{M}_{i}, \quad i=0, \ldots, m-1, \quad r<1
$$

и неравенств для коэффициентов $b_{k i}$ :

$$
\sum_{k=1}^{\infty}\left(b_{k i}\left(1-r^{2}\right)^{i} r^{k}\right)^{2} \leqslant \mathscr{M}_{i}, \quad i=0, \ldots, m-1, \quad r<1
$$

Покажем, что из (49) вытекают неравенства (50); доказательство того, что из $\left(49^{\prime}\right)$ вытекают неравенства в $\left(50^{\prime}\right)$, совершенно аналогично.

Возьмем произвольное $N \geqslant 1$. Из (49) имеем неравенство

$$
\sum_{k=1}^{N}\left(\sum_{i=0}^{m-1} a_{k i}\left(1-r^{2}\right)^{i} r^{k}\right)^{2} \leqslant \mathscr{M}, \quad r<1
$$

после перехода в котором к пределу при $r \rightarrow 1$, получим

$$
\sum_{k=1}^{N} a_{k 0}^{2} \leqslant \mathscr{M} \text { при любом } N \geqslant 1
$$

т.е. имеет место неравенство $\sum_{k=1}^{\infty} a_{k 0}^{2} \leqslant \mathscr{M}$, а значит, и неравенство

$$
\sum_{k=1}^{\infty} a_{k 0}^{2} r^{2 k} \leqslant \mathscr{M}, \quad r<1
$$

которое совпадает с нулевьп (при $i=0)$ неравенством в $(50)$. 
Из неравенства (49) и доказанного для $i=0$ неравенства в (50) вытекает, что имеет место неравенство

$$
\sum_{k=1}^{\infty}\left(\sum_{i=1}^{m-1} a_{k i}\left(1-r^{2}\right)^{i} r^{k}\right)^{2} \leqslant \mathscr{M}^{\prime}, \quad r<1, \quad \mathscr{M}^{\prime}=\text { const }
$$

В случае $m=2$, т.е. для того случая, когда уравнение в (1) бигармоническое, лемма 7 установлена, так как неравенство (51) в этом случае совпадает со вторьм (и последним) неравенством в (50). Поэтому в дальнейшем считаем, что $m>2$.

Нам удобнее иметь дело не с неравенством (51), а с полученным из него, как и при доказательстве леммы 3, с помощью замены переменной $1-r^{2}=x, x \in(0,1)$; $x \rightarrow 0$ при $r \rightarrow 1$, неравенством

$$
\sum_{k=1}^{\infty}\left(\sum_{i=1}^{m-1} a_{k i} x^{i}(1-x)^{k / 2}\right)^{2} \leqslant \mathscr{M}^{\prime}, \quad x \in(0,1) .
$$

Из этого неравенства нам нужно получить оставшиеся в (50) недоказанными неравенства для $i=1, \ldots, m-1$. Из (52) вытекает, что при произвольном целом $N \geqslant 1$ имеет место неравенство

$$
\sum_{(N, 2 N]}\left(\sum_{i=1}^{m-1} a_{k i} x^{i-1}(1-x)^{k / 2}\right)^{2} \leqslant \frac{\mathscr{M}^{\prime}}{x^{2}}, \quad x \in(0,1)
$$

проинтегрировав которое по $x$ по интервалу $(x, 1)$, получим

$$
\sum_{(N, 2 N]} \int_{x}^{1}\left(\sum_{i=1}^{m-1} a_{k i} \xi^{i-1}(1-\xi)^{k / 2}\right)^{2} d \xi \leqslant \frac{\mathscr{M}^{\prime}(1-x)}{x}, \quad x \in(0,1) .
$$

Полагая в последнем неравенстве $N=1 / x$ и считая $x$ достаточно малым, $x \in$ $\left(0, \frac{1}{3}\right)$, получим

$$
\sum_{\left(\frac{1}{x}, \frac{2}{x}\right]} \frac{x}{1-x} \int_{x}^{1}\left(\sum_{i=1}^{m-1} a_{k i} \xi^{i-1}(1-\xi)^{k / 2}\right)^{2} d \xi \leqslant \mathscr{M}^{\prime}, \quad x \in\left(0, \frac{1}{3}\right)
$$

Введем обозначения

$$
\frac{a_{k i}}{k^{i}}=\alpha_{k i}, \quad k \geqslant 1, \quad i=1, \ldots, m-1 ;
$$

тогда последнее неравенство перепишется в виде

$$
\sum_{\left(\frac{1}{x}, \frac{2}{x}\right]} \frac{k^{2} x}{1-x} \int_{x}^{1}\left(\sum_{i=1}^{m-1} \alpha_{k i}(k \xi)^{i-1}(1-\xi)^{k / 2}\right)^{2} d \xi \leqslant \mathscr{M}^{\prime}, \quad x \in\left(0, \frac{1}{3}\right),
$$

или в виде

$$
\sum_{\left(\frac{1}{x}, \frac{2}{x}\right]} \frac{k x}{1-x} \int_{k x}^{k}\left(\sum_{i=1}^{m-1} \alpha_{k i} \xi^{i-1}\left(1-\frac{\xi}{k}\right)^{k / 2}\right)^{2} d \xi \leqslant \mathscr{M}^{\prime}
$$


Рассмотрим функцию

$$
\mathscr{P}\left(x, k, \alpha_{1}, \ldots, \alpha_{m-1}\right)=\frac{k x}{1-x} \int_{k x}^{k}\left(\sum_{i=1}^{m-1} \alpha_{i} \xi^{i-1}\left(1-\frac{\xi}{k}\right)^{k / 2}\right)^{2} d \xi
$$

очевидно, неотрицательную и непрерывную на (неограниченном) множестве $Q=\left\{0<x \leqslant \frac{1}{3}, 1 \leqslant k x \leqslant 2, \alpha_{1}^{2}+\cdots+\alpha_{m-1}^{2}=1\right\}$. Покажем, что (неотрицательная) точная нижняя грань этой функции по множеству $Q$ положительна:

$$
\lambda_{1}=\inf _{Q} \mathscr{P}>0
$$

Предположим, напротив, что $\lambda_{1}=0$. Прежде всего, заметим, что неможет быть конечной точки $\left(x_{0}, k_{0}, \alpha_{1}^{0}, \ldots, \alpha_{m-1}^{0}\right), x_{0}>0$, из $Q$, в которой $\mathscr{P}=0$ : наличие такой точки приводило бы к равенству

$$
\frac{k_{0} x_{0}}{1-x_{0}} \int_{k_{0} x_{0}}^{k_{0}}\left(\sum_{i=1}^{m-1} \alpha_{i}^{0} \xi^{i-1}\left(1-\frac{\xi}{k_{0}}\right)^{k_{0} / 2}\right)^{2} d \xi=0
$$

которое возможно только при $\alpha_{i}^{0}=0, i=1, \ldots, m-1$. Следовательно, из предположения $\lambda_{1}=0$ вытекает существование последовательностей $x_{n}, n=1,2, \ldots$, $k_{n}, n=1,2, \ldots, \alpha_{1}^{n}, n=1,2, \ldots, \alpha_{m-1}^{n}, n=1,2, \ldots, x_{n} \rightarrow 0, k_{n} \rightarrow \infty$ при $n \rightarrow \infty$, для которых

$$
\frac{k_{n} x_{n}}{1-x_{n}} \int_{k_{n} x_{n}}^{k_{n}}\left(\sum_{i=1}^{m-1} \alpha_{i}^{n} \xi^{i-1}\left(1-\frac{\xi}{k_{n}}\right)^{k_{n} / 2}\right)^{2} d \xi \rightarrow 0, \quad n \rightarrow \infty,
$$

и тем более (поскольку $2 k_{n} x_{n}<k_{n}$ )

$$
\frac{k_{n} x_{n}}{1-x_{n}} \int_{k_{n} x_{n}}^{2 k_{n} x_{n}}\left(\sum_{i=1}^{m-1} \alpha_{i}^{n} \xi^{i-1}\left(1-\frac{\xi}{k_{n}}\right)^{k_{n} / 2}\right)^{2} d \xi \rightarrow 0, \quad n \rightarrow \infty .
$$

За счет, быть может, прореживания этих последовательностей их можно считать дополнительно обладающими следующими свойствами: $x_{n} k_{n} \rightarrow \theta, n \rightarrow \infty$, где $\theta$ - некоторое число отрезка $[1,2]$ :

$$
1 \leqslant \theta \leqslant 2 ; \quad \alpha_{1}^{n} \rightarrow \alpha_{1}^{0}, n \rightarrow \infty ; \ldots ; \alpha_{m-1}^{n} \rightarrow \alpha_{m-1}^{0}, n \rightarrow \infty ;
$$

$\left(\alpha_{1}^{0}\right)^{2}+\cdots+\left(\alpha_{m-1}^{0}\right)^{2}=1$. Это означает, что должно выполняться равенство

$$
\theta \int_{\theta}^{2 \theta}\left(\sum_{i=1}^{m-1} \alpha_{i}^{0} \xi^{i-1} e^{-\xi / 2}\right)^{2} d \xi=0
$$

которого быть не может. Неравенство (56) установлено.

Функция $\mathscr{P}\left(x, k, \alpha_{1}, \ldots, \alpha_{m-1}\right)$, (см. (55)), является квадратичной формой по переменньм $\alpha_{1}, \ldots, \alpha_{m-1}$

$$
\mathscr{P}\left(x, k, \alpha_{1}, \ldots, \alpha_{m-1}\right)=\sum_{i, j=1}^{m-1} A_{i j}(x, k) \alpha_{i} \alpha_{j}
$$


где

$$
A_{i j}(x, k)=\frac{k x}{1-x} \int_{k x}^{k} \xi^{i+j-1}\left(1-\frac{\xi}{k}\right)^{k} d \xi, \quad i, j=1, \ldots, m-1 .
$$

При всех интересующих нас значениях $(x, k), 0<x \leqslant \frac{1}{3}, 1 \leqslant k x \leqslant 2$, симметрическая матрица $\left\|A_{i j}(x, k)\right\|$ положительно определена и ее минимальное собственное значение совпадает с числом $\lambda_{1}$ из (56). Следовательно, в силу (54) для всех $x \in\left(0, \frac{1}{3}\right)$

$$
\lambda_{1} \sum_{(1 / x, 2 / x]} \sum_{i=1}^{m-1} \alpha_{k i}^{2} \leqslant \sum_{(1 / x, 2 / x]} \frac{k x}{1-x} \int_{k x}^{k}\left(\sum_{i=1}^{m-1} \alpha_{k i} \xi^{i-1}\left(1-\frac{\xi}{k}\right)^{k / 2}\right)^{2} d \xi \leqslant \mathscr{M}^{\prime},
$$

откуда согласно с определением (53) имеем при достаточно больших $N$

$$
\sum_{(N, 2 N]} \frac{a_{k i}^{2}}{k^{2 i}} \leqslant \frac{\mathscr{M}^{\prime}}{\lambda_{1}} \text { при каждом } i, \quad i=1, \ldots, m-1 .
$$

Неравенства в (50) для $i=1, \ldots, m-1$ теперь вытекают из леммы 3. Лемма 7 доказана.

ЛЕмма 8. Если существует $L_{2}$-предел на границе $\{|x|=1\}$ функиии

$$
U(x)=\left(1-|x|^{2}\right) u_{1}(x)+\cdots+\left(1-|x|^{2}\right)^{m-1} u_{m-1}(x), \quad|x|<1, \quad m>1,
$$

где $u_{1}(x), \ldots, u_{m-1}(x)$ - гармонические функиии в круге $\{|x|<1\}$, то этот предел равен нулю.

ЗАмЕчАнИЕ. Утверждение леммы 8, в частности, справедливо, конечно, и для функции $U(x)$, равной какому-нибудь одному слагаемому из правой части (58): $U(x)=\left(1-|x|^{2}\right)^{i} u_{i}(x), i=1, \ldots, m-1$.

ДокАЗАТЕЛЬСТво ЛЕммы. Пусть $f(\varphi)-L_{2}$-предел функции $U(x)$ при $r \rightarrow 1$ и пусть разложения в ряды Фурье функции $f(\varphi)$ и функций $u_{i}(r, \varphi), i=1, \ldots, m-1$, задаются формулами (7) и (14). Тогда разложение в ряд Фурье функции $U(r, \varphi)$ имеет вид

$$
\begin{aligned}
U(r, \varphi)= & \sum_{i=1}^{m-1} \frac{a_{0 i}}{2}\left(1-r^{2}\right)^{i}+\sum_{k=1}^{\infty}\left(\sum_{i=1}^{m-1} a_{k i} r^{k}\left(1-r^{2}\right)^{i}\right) \cos k \varphi \\
& +\sum_{k=1}^{\infty}\left(\sum_{i=1}^{m-1} b_{k i} r^{k}\left(1-r^{2}\right)^{i}\right) \sin k \varphi .
\end{aligned}
$$

Из $L_{2}$-сходимости вытекает слабая сходимость. Следовательно, коэффициенты Фурье функции $f$ являются пределами при $r \rightarrow 1$ соответствующих коэффициентов функции $U$ :

$$
\begin{aligned}
a_{k} & =\lim _{r \rightarrow 1} \sum_{i=1}^{m-1} a_{k i} r^{k}\left(1-r^{2}\right)^{i}=0, \quad i=0,1, \ldots, \\
b_{k} & =\lim _{r \rightarrow 1} \sum_{i=1}^{m-1} b_{k i} r^{k}\left(1-r^{2}\right)^{i}=0, \quad i=1,2, \ldots
\end{aligned}
$$

Следовательно, $f=0$. Лемма доказана. 
ЛЕмма 9. т-гармоническая функиия $u(x),|x|<1$, имеет $L_{2}$-предел на границе $\{|x|=1\}$ тогда и только тогда, когда имеют $L_{2}$-предель на границе все слагаемые $\left(1-|x|^{2}\right)^{i} u_{i}(x), i=0, \ldots, m-1$, правой части равенства $(10)$.

В доказательстве нуждается лишь утверждение, что из существования $L_{2}$-предела на гранище функции $u(x)$ вытекает существование $L_{2}$-пределов на границе функций $\left(1-|x|^{2}\right)^{i} u_{i}(x), i=0, \ldots, m-1$.

Пусть $L_{2}$-предел на границе функции $u(x)$ существует. Тог да она $L_{2}$-ограничена, а по лемме $7 L_{2}$-ограничена и функция $u_{0}(x)$, которая, тем самым, являясь решением эллиптического уравнения второго порядка, имеет $L_{2}$-предел на границе. Следовательно, $L_{2}$-предел на границе имеет и функция $u(x)-u_{0}(x)$, являющаяся функцией $U(x)$, вида $(58)$. Согласно лемме 8 этот $L_{2}$-предел равен нулю:

$$
\|U(r, \cdot)\|_{L_{2}} \rightarrow 0, \quad r \rightarrow 1
$$

Из формулы (59) тогда немедленно вытекает, что имеет место соотношение

$$
\sum_{k=1}^{\infty}\left(\sum_{i=1}^{m-1} a_{k i}\left(1-r^{2}\right)^{i} r^{k}\right)^{2} \rightarrow 0, \quad r \rightarrow 1
$$

и соответствующее соотношение, связанное с коэффициентами $b_{k i}$ :

$$
\sum_{k=1}^{\infty}\left(\sum_{i=1}^{m-1} b_{k i}\left(1-r^{2}\right)^{i} r^{k}\right)^{2} \rightarrow 0, \quad r \rightarrow 1
$$

Поэтому по произвольному $\varepsilon>0$ найдется такое $r_{0}<1$, что для всех $r, r_{0} \leqslant r<1$, имеет место неравенство

$$
\sum_{k=1}^{\infty}\left(\sum_{i=1}^{m-1} a_{k i}\left(1-r^{2}\right)^{i} r^{k}\right)^{2} \leqslant \varepsilon
$$

Это означает (см. доказательство леммы 7 ), что для всех $x, 0<x \leqslant x_{0}$, где $x_{0}=1-r_{0}^{2}$, имеет место неравенство (52) с $\mathscr{M}^{\prime}=\varepsilon$ и, тем самым, в силу $(57)$

$$
\sum_{(N, 2 N]} \frac{a_{k i}^{2}}{k^{2 i}} \leqslant \frac{\varepsilon}{\lambda_{1}} \text { при каждом } i=1, \ldots, m-1,
$$

для всех достаточно больших $N$. Аналогично, конечно, получаем и неравенства

$$
\sum_{(N, 2 N]} \frac{b_{k i}^{2}}{k^{2 i}} \leqslant \frac{\varepsilon}{\lambda_{1}} \text { при каждом } i=1, \ldots, m-1,
$$

справедливые для всех достаточно больших $N$. То есть

$$
\sum_{(N, 2 N]} \frac{a_{k i}^{2}+b_{k i}^{2}}{k^{2 i}} \rightarrow 0 \text { при } N \rightarrow \infty .
$$

Из леммы 7 после этого вытекает, что $\left\|\left(1-r^{2}\right)^{i} u_{i}(r, \cdot)\right\|_{L_{2}} \rightarrow 0$ при $r \rightarrow 1$ для любого $i=1, \ldots, m-1$. Лемма доказана.

Вполне аналогично доказываются и последние две леммы. 
ЛЕмма 10. т-гармоническая в круге $\{|x|<1\}$ функция $u(x)$ удовлетворяет условию

$$
\sup _{r<1}\left\|P_{N} u(r, \cdot)\right\|_{L_{2}} \rightarrow 0 \quad \text { npu } \quad N \rightarrow \infty
$$

тогда и только тогда, когда этому условию удовлетворяют все слагаемые правой части равенства (10):

$$
\sup _{r<1}\left\|P_{N}\left(1-r^{2}\right)^{i} u_{i}(r, \cdot)\right\|_{L_{2}} \rightarrow 0 \quad \text { npu } \quad N \rightarrow \infty
$$

для всех $i=0,1, \ldots, m-1$.

ЛЕмма 11. т-гармоническая в круге $\{|x|<1\}$ функция $и(x)$ удовлетворяет условию

$$
\sup _{r<1}\left\|\frac{\delta_{h}^{2} u(r, \cdot)}{h}\right\|_{H^{-1}} \rightarrow 0 \quad \text { npu } \quad h \rightarrow 0
$$

тогда и только тогда, когда этому условию удовлетворяет каждое слагаемое правой части равенства (10):

$$
\sup _{r<1}\left\|\frac{\delta_{h}^{2}\left(1-r^{2}\right)^{i} u_{i}(r, \cdot)}{h}\right\|_{H^{-1}} \rightarrow 0 \quad \text { npu } \quad h \rightarrow 0
$$

для всех $i=0, \ldots, m-1$.

ДоКАЗАТЕЛЬСТВо ЛЕММЫ 10. Из формул (10) и (48)

$P_{N} u(r, \varphi)=\sum_{(N, 2 N]}\left(\sum_{i=0}^{m-1} a_{k i} r^{k}\left(1-r^{2}\right)^{i}\right) \cos k \varphi+\sum_{(N, 2 N]}\left(\sum_{i=0}^{m-1} b_{k i} r^{k}\left(1-r^{2}\right)^{i}\right) \sin k \varphi$

и, тем самым, по условию леммы 10 (в доказательстве нуждается, конечно, лишш утверждение о необходимости) по любому $\varepsilon>0$ найдется такое $N_{0}>0$, что

$$
\varepsilon \geqslant \sum_{(N, 2 N]}\left(\sum_{i=0}^{m-1} a_{k i} r^{k}\left(1-r^{2}\right)^{i}\right)^{2} \text { для всех } r<1 \text { и } N \geqslant N_{0} .
$$

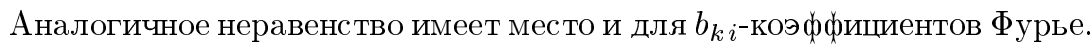

Положим сначала, как и при доказательстве леммы 7 , в формуле (62) $r$ равньм единице. В результате получим неравенство

$$
\sum_{(N, 2 N]} a_{k 0}^{2} r^{2 k} \leqslant \sum_{(N, 2 N]} a_{k 0}^{2} \leqslant \varepsilon \text { для всех } r<1 \text { и всех } N \geqslant N_{0},
$$

из которого вытекает доказываемое соотношение (60) для $i=0$ и неравенство

$$
\sum_{(N, 2 N]}\left(\sum_{i=1}^{m-1} a_{k i} r^{k}\left(1-r^{2}\right)^{i}\right)^{2} \leqslant 4 \varepsilon \text { для всех } r<1 \text { и } N \geqslant N_{0}
$$


или, что то же, неравенство

$$
\sum_{(N, 2 N]}\left(\sum_{i=1}^{m-1} a_{k i} x^{i}(1-x)^{k / 2}\right)^{2} \leqslant 4 \varepsilon \text { для всех } x \in(0,1) \text { и } N \geqslant N_{0}
$$

(сделана замена переменной $1-r^{2}=x$ ).

Как и при доказательстве леммы 7 , проделаем с последним неравенством последовательно следующие операции: разделим на $x^{2}$, проинтегрируем по интервалу $(x, 1)$, умножим на $x /(1-x)$ и положим $x=1 / N$ (считая, что $x$ - достаточно мало). В результате после введения обозначений: $a_{k i} / k^{i}=\alpha_{k i}, k \geqslant 1, i=1, \ldots, m-1$, получим неравенство

$$
\sum_{(1 / x, 2 / x]} \frac{x k}{1-x} \int_{k x}^{k}\left(\sum_{i=1}^{m-1} \alpha_{k i} \xi^{i-1}\left(1-\frac{\xi}{k}\right)^{k / 2}\right)^{2} d \xi \leqslant 4 \varepsilon
$$

совпадающее с неравенством $(54)$, в котором $\mathscr{M}^{\prime}=4 \varepsilon$. Следовательно, имеют место неравенства $(57)$ с $\mathscr{M}^{\prime}=4 \varepsilon$ и аналогичные неравенства для $b_{k i}$-коэффициентов Фурье. А тогда на основании лемм 3 и 6 получаем оставшиеся еще недоказанньми для $i=1, \ldots, m-1$ соотношения в (60). Лемма 10 доказана.

ДокАЗАТЕЛЬСтво ЛЕммы 11. Согласно (10) и (48)

$$
\begin{aligned}
\frac{\delta_{h}^{2} u(r, \varphi)}{h}= & \sum_{k=1}^{\infty}\left(\sum_{i=0}^{m-1} a_{k i}\left(1-r^{2}\right)^{i} r^{k}\right) \frac{1-\cos k h}{h} \cos k \varphi \\
& +\sum_{k=1}^{\infty}\left(\sum_{i=0}^{m-1} b_{k i}\left(1-r^{2}\right)^{i} r^{k}\right) \frac{1-\cos k h}{h} \sin k \varphi
\end{aligned}
$$

поэтому по условию леммы 11 (в ней мы тоже, естественно, доказываем лишь утверждение о необходимости) по любому $\varepsilon>0$ найдется такое $h_{0}>0$, что для всех $h \leqslant h_{0}$ и всех $r<1$

$$
\sum_{k=1}^{\infty}\left(\sum_{i=0}^{m-1} a_{k i}\left(1-r^{2}\right)^{i} r^{k}\right)^{2}\left(\frac{1-\cos k h}{h}\right)^{2} \leqslant \varepsilon
$$

(аналогичное неравенство имеет место и для $b_{k i}$-коэффициентов Фурье). Так же, как и при доказательстве леммы 7 , убеждаемся, что для всех $r<1$ и всех $h \leqslant h_{0}$

$$
\sum_{k=1}^{\infty} a_{k 0}^{2} r^{2 k}\left(\frac{1-\cos k h}{k h}\right)^{2} \leqslant \varepsilon
$$

Это означает, что имеет место соотношение (61) для $i=0$. Кроме того, из последних двух неравенств вытекает неравенство

$$
\sum_{k=1}^{\infty}\left(\sum_{i=1}^{m-1} a_{k i}\left(1-r^{2}\right)^{i} r^{k}\right)^{2}\left(\frac{1-\cos k h}{k h}\right)^{2} \leqslant 4 \varepsilon
$$


для всех $r<1$ и всех $h \leqslant h_{0}$,

$$
\sum_{(N, 2 N]} \frac{a_{k i}^{2}+b_{k i}^{2}}{k^{2 i}}\left(\frac{1-\cos k h}{k h}\right)^{2} \leqslant \frac{4 \varepsilon}{\lambda_{1}}, \quad i=1, \ldots, m-1
$$

(неравенства для $b_{k i}$-коэффициентов, как уже неоднократно отмечалось, получаются в полной аналогии с соответствующими неравенствами для $a_{k i}$-коэффициентов). Поскольку

$$
\frac{\sin ^{4} \varphi}{\varphi^{2}} \geqslant \min \left\{\frac{\sin ^{4} \frac{1}{2}}{\frac{1}{4}}, \frac{\sin ^{4} \frac{5}{2}}{\frac{25}{4}}\right\}=\gamma^{\prime}>0
$$

при $\varphi \in\left(\frac{1}{2}, \frac{5}{2}\right)$, то

$$
\left(\frac{1-\cos k h}{k h}\right)^{2}=\left(\frac{\sin ^{2} \frac{k h}{2}}{\frac{k h}{2}}\right)^{2} \geqslant \gamma^{\prime} \text { при всех (целых) } k \in\left(\frac{1}{h}, \frac{5}{h}\right) .
$$

Следовательно, полагая в неравенствах (63) $h$ равным $2 / N$, при достаточно больших $N\left(N \geqslant 2 / h_{0}\right)$ и учитьвая, что $[N, 2 N] \subset\left(\frac{1}{2} N, \frac{5}{2} N\right)$, получим, что имеют место неравенства

$$
\sum_{(N, 2 N]} \frac{a_{k i}^{2}+b_{k i}^{2}}{k^{2 i}} \leqslant \frac{4 \varepsilon}{\lambda_{1} \gamma^{\prime}}
$$

для всех достаточно больших $N$ и всех $i=1, \ldots, m-1$, т.е.

$$
\sum_{(N, 2 N]} \frac{a_{k i}^{2}+b_{k i}^{2}}{k^{2 i}} \rightarrow 0 \text { при } N \rightarrow \infty \text { для всех } i=1, \ldots, m-1 .
$$

Применяя после этого леммы 3 и 6 , получим недостающие при $i=1, \ldots, m-1$ соотношения в (61). Лемма 11 доказана.

\section{§2. Доказательства теорем}

Теоремы 1 и 2 будем доказывать одновременно.

Необходимость. Пусть сушествует $L_{2}$-предел на границе $m$-гармонической функции $u(x)$. Тогда $u(x) L_{2}$-ограничена, т.е. вьполняются первые условия доказываемых теорем, и по лемме $7 L_{2}$-ограничена функция $u_{0}(x)$ из равенства $(10)$. По лемме 2 функция $u_{0}(x)$ удовлетворяет условиям

$$
\sup _{r<1}\left\|P_{N} u_{0}(r, \cdot)\right\|_{L_{2}} \rightarrow 0 \text { при } N \rightarrow \infty
$$

и

$$
\sup _{r<1}\left\|\frac{\delta_{h}^{2} u_{0}(r, \cdot)}{h}\right\|_{H^{-1}} \rightarrow 0 \text { при } h \rightarrow 0 .
$$

По лемме 9 каждое слагаемое $\left(1-|x|^{2}\right)^{i} u_{i}(x), i=1, \ldots, m-1$, правой части формулы (10) имеет предел, совпадающий по лемме 8 с нулевой функцией. Следовательно, по лемме 6

$$
\sup _{r<1}\left\|P_{N}\left(1-r^{2}\right)^{i} u_{i}(r, \cdot)\right\|_{L_{2}} \rightarrow 0 \text { при } N \rightarrow \infty, \quad i=1, \ldots, m-1,
$$


И

$$
\sup _{r<1}\left\|\frac{\delta_{h}^{2}\left(1-r^{2}\right)^{i} u_{i}(r, \cdot)}{h}\right\|_{H^{-1}} \rightarrow 0 \text { при } h \rightarrow 0, \quad i=1, \ldots, m-1 .
$$

Поэтому функция $u(x)$ удовлетворяет и вторым условиям доказываемых теорем.

Достаточность. Пусть $m$-гармоническая функция $u(x) L_{2}$-ограничена, и для нее выполняются условие (8) или условие (9). Докажем, что она имеет $L_{2}$-предел на границе. По лемме 7 функция $u_{0}(x)$ из представления $(10) L_{2}$-ограничена; следовательно, существует ее $L_{2}$-предел на гранище. Из вторых условий доказываемых теорем согласно леммам 10 и 11 соответственно имеют место для всех $i=1, \ldots, m-1$ соотношения (64) или (65). Но тогда по лемме 6 существуют (нулевые) $L_{2}$-пределы на границе слагаемых $\left(1-|x|^{2}\right)^{i} u_{i}(x), i=1, \ldots, m-1$. Следовательно, функция $u(x)$ имеет $L_{2}$-предел на границе. Теоремы 1 и 2 доказаны.

В теореме 3 в доказательстве нуждается лишь утверждение о достаточности. В силу критерия компактности в $L_{2}$ множество $u(r, \varphi), r<1$, равномерно $L_{2}$-ограничено, т.е. выполняется условие $(2)$, и $L_{2}$-равностепенно непрерывно, т.е. вьполняется условие

$$
\sup _{r<1}\left\|\delta_{h}^{1} u(r, \cdot)\right\|_{L_{2}} \rightarrow 0 \quad \text { при } \quad h \rightarrow 0
$$

где $\delta_{h}^{1} u(r, \varphi)=u(r, \varphi+h)-u(r, \varphi)$, из которого в силу очевидного неравенства

$$
\left\|\delta_{h}^{1} u(r, \cdot)\right\|_{L_{2}} \geqslant\left\|\frac{\delta_{h}^{2} u(r, \cdot)}{h}\right\|_{H^{-1}}, \quad h \neq 0
$$

вытекает условие (9). Таким образом, теорема 3 следует из теоремы 2.

\section{Список литературы}

1. Ройтберг Я.A. О граничных значениях обобщенных решений эллиптических уравнений // ДАН СССР. 1969. Т. 188. №1. С. 41-44.

2. Мусхелишвили Н. И. Некоторые основныезадачи математической теории упругости. М.: Наука, 1966.

Математический институт

Поступила в редакцию

им. В.А. Стеклова РАН

20.06.1996 